\title{
Notes on Operations IGAPS: A Taxonomy and Facet Classification System
}

\author{
John Pell and Meghan Huppuch
}

This paper describes an assessment of the information management practices at International Planned Parenthood Federation/Western Hemisphere Region and the development and implementation of an information management pilot for that organization. The pilot included the development of a taxonomy to classify the organization's documents, training in basic citation practices, and a decentralized model for building an organized library of documents within the citation management software Mendeley. The authors discuss the pilot's taxonomy within the context of literature on taxonomy development and offers strategic recommendations for improving the information management practices of not-for-profit organizations that lack dedicated information management staff.

T nternational Planned Parenthood Federation/Western Hemisphere Region 1 (IPPF/WFR), the not-for-profit organization that sponsored this project phased out its library in 2011 when the librarian it employed left to accept another position. The organization did not seek a replacement, rationalizing that the information it needed for research and publications was easily accessible online for free. The organization's communications and evaluations officers soon noticed the development of difficulties with the retrieval of research material and problems with complete and accurate citation in organizational publications. Accordingly, they suggested the following two main objectives for this project: 1) develop a taxonomy to facilitate access to journal and grey literature, and 2) train staff to use of the taxonomy and citation management software to increase their ability to systematically document, track, and locate citations of data and literature in speeches, presentations, papers, and internal publications. The organization's communications and evaluations officers tasked a temporary information management specialist to carry out these objectives. An academic librarian provided pro bono consultation and training for the communications and evaluations officers and information management specialist. This paper provides an overview of the literature on taxonomy development that informed the approach to taxonomy development taken at the organization, a description of the methods used to assess the need for information management at the organization, and a discussion of the results of the project.

John Pell (jpell@hunter.cuny.edu) is an Assistant Professor at Hunter College Library in New York, New York. Meghan Huppuch (meghan.huppuch@gmail .com) is a communications professional at a philanthropic foundation.

Manuscript submitted August 4, 2016: returned to authors for revision September 20, 2016; revised manuscript submitted November 16, 2016; manuscript accepted for publication March 1, 2017.

\section{Literature Review}

A search of Library and Information Science Source, Library and Information Science Abstracts, Business Source Premier, and the Directory of Open access journals retrieved 924 papers published between 2004 and 2015 that include the word "taxonomy" in the title; none of these papers described developing a taxonomy for use within a citation manager. After excluding papers that were primarily descriptions of taxonomies in domains unrelated to women's reproductive health or that described automated methods of taxonomy production based on 
inaccessible tools, the search yielded twenty-two papers that dealt explicitly with methods of taxonomy creation or assessment, or description of taxonomies related to the domain of women's health. This review uses those papers to accomplish three functions: define the features of taxonomies, describe methods of producing taxonomies, and analyze the costs and benefits of using and creating taxonomies.

\section{Defining Taxonomy}

A taxonomy is commonly understood as a system of knowledge organization that is closely related to the practice of classification. Confusion may ensue in discussions of taxonomy as systems for knowledge organization are described variously in the literature of information science and business as classifications, frameworks, typologies, taxonomies, and ontologies that may vary in the complexity of their structure from flat lists of terms to densely interconnected hierarchies with arrays of branching subcategories. ${ }^{1}$

Some of these differences in description are interpreted as different approaches to analysis of the same underlying relationships. For example, in the National Library of Medicine's (NLM) Medical Subject Heading (MeSH) database, "classification" is listed as a child element, or subcategory, of both "information science" and "documentation." A taxonomist could represent the relationships among these terms in the form of a network in which "classification" has multiple parent elements. Alternatively, a taxonomist could represent "classification" as existing in multiple hierarchical lists. Nickerson, Varshney, and Munterman describe taxonomies as defined sets of objects. These objects have dimensions and the dimensions in turn have defined characteristics. They posit two restrictions on taxonomies requiring that characteristics be mutually exclusive, meaning that each dimension should have exactly one characteristic, and collectively exhaustive, meaning that each object should have a characteristic for every one of its dimensions. They explicitly invoke Miller's "Magical Number Seven, Plus or Minus Two" as a possible objective criteria related to their requirement that a taxonomy be concise. ${ }^{2}$ Neelameghan and Rhagavan extend this argument for the significance of Miller's number in classification schemes through a broad overview of knowledge organization drawing examples from Vedanta philosophy, religious mysticism, and modern approaches to systems development; they note that prominent classification systems that persist over time have between five and nine top-level categories. ${ }^{3}$

A taxonomy may be as simple as a list of terms, but that does not imply it is neutral with regard to social values or theoretical perspectives. Classification can raise ethical issues when determinations about the scientific merit of materials are in question, such as when a college library director was asked to classify creationist materials as "science" rather than "religion" in the college's library. ${ }^{4}$ A theoretical perspective may drive taxonomy development and help to further the development of a field, as McKinney and Yoos intended their taxonomy of views on information to help advance the field of information science. ${ }^{5}$

While agreement about what is meant by "taxonomy" may further the development or unification of a discipline, differences in perspective or purpose about a taxonomy's entities may divide disciplines. A historic example of this type of difference of perspective is found in the phrenetic and cladistic approaches to taxonomy development in systematics: the phrenetic approach groups organisms on the basis of shared characteristics and the cladistic approach groups organisms on the basis of shared ancestry. The phrenetic approach dominated when physical observation was the primary research method in the field, but as techniques for analyzing genetic distance emerged, cladistic taxonomies were developed to focus on the common ancestry of group of organisms. The schism between scientists using these two taxonomies engendered not only scientific debates, but also impassioned disputes over bias toward one form of taxonomy or the other in the editorship of scientific journals. ${ }^{6}$

Another more recent example is from scientometrics in the form of taxonomic disputes over citation metrics. Bornman follows Kuhn's theory to characterize scientific revolutions as taxonomic changes in a research field and proposes that such a revolution is currently underway in the field of scientometrics. ${ }^{7}$

If the relatively stolid fields of systematics and scientometrics are vulnerable to controversy and upheaval through disputes over taxonomy, a taxonomist would be well advised to tread carefully in a field that carries more politicized controversy, such as reproductive health. In her taxonomy and framing analysis of abortion weblogs, Park distinguishes between advocacy versus objectivist framings of the blog posts. ${ }^{8}$ While such distinctions may be made with relative ease academically, considering research that suggests perceptions of objectivity may vary with an individual's political bias, classifying information as advocacy or objectivity could prove controversial in an organization with politically diverse views, or it could seem to have limited usefulness for an organization that views its purpose as closely aligned with advocacy. ${ }^{9}$

Despite the controversies that may ensue, theoretical basis is not necessarily a vulnerability to avoid in taxonomy development; rather, it may be a goal to strive toward. As taxonomic classifications can serve as the basis for organizational decision-making, it is important that those classifications are based in meaningful characteristics. Tezanos, Vazquez, and Sumner criticize the use of per capita income as the criteria for defining developing nations because such classification lacks a sound theoretical basis. They point out 
that, even absent a developed and agreed upon theory, a multidimensional taxonomy that takes more entities and characteristics into account will provide greater utility for decision-making and theory development. ${ }^{10}$

Additionally, taxonomies may differ in more pragmatic terms regarding whether they classify their objects by physical characteristics, historical origins, or intended uses. User intent for interacting with a taxonomic entity is often an important consideration in taxonomy development. The RoMEO taxonomy for copyright transfer agreements categorizes agreements based on which format of publication an author may distribute and how and to where the author may distribute it. ${ }^{11}$

Doria's theoretical taxonomy of document uses included eight categories: individual work document, collaborative work document, project monitoring document, trade document, auxiliary resource document, referential document, external document, and record document. While Doria noted that this theoretical taxonomy could be applied to any department in an organization, her empirically developed taxonomy of a document collection from an engineering firm's research department produced fifty-seven categories, including budget, needs analysis, and case scenario; although Doria's theoretical categories promised potential, they required significant modification to meet the needs of actual users within a specific context. ${ }^{12}$

\section{Taxonomy Development Methods}

Nickerson, Varshney, and Munterman reported that over forty of the papers that they surveyed in their review of taxonomy development literature did not report a methodology for taxonomy development. Reports that described a method classified it as inductive, deductive, or intuitive, and observed that nearly a third of these reports used an intuitive, or ad hoc, approach to develop the taxonomy. ${ }^{13}$

As an alternative to ad hoc approaches, Nickerson, Varshney, and Munterman present a four-stage model of taxonomy development that incorporates recursive processes. This model begins with the determination of a "metacharacteristic" that serves to dictate the characteristics to be included in the taxonomy. Following the determination of the meta-characteristic, ending conditions for the development of the taxonomy are set. Development then proceeds through recursive stages of identification, modification, and evaluation until the previously defined ending conditions are met. Even the meta-characteristic may not clearly emerge until multiple iterations of the development approach have conducted.

In its study of the Functional Requirements of Bibliographic Records (FRBR), the International Federation of Library Associations and Institutions (IFLA) applied an entity analysis technique. At a basic level, entity analysis consists of isolating the entities of interest to users in a particular domain. These entities are defined in a way that focuses on the entities themselves, rather than the data about them. ${ }^{14}$ An example from the domain of reproductive health would be women with cervical cancer, as opposed to statistics about women with cervical cancer. After entities are defined, each entity's characteristics may be enumerated. This method of entity analysis may be extended to include relationships between entities and user tasks.

In a paper outlining the basic phases and best practices of taxonomy development, Cisco identifies the four basic phases of taxonomy development as planning and analysis; design, development, and testing; implementation; and maintenance. Cisco's best practice are: keeping the taxonomy closely related to the organizational strategy, incorporating existing taxonomy and metadata, making categories well-defined and distinct, developing the taxonomy in an iterative process, and providing for adequate resources to maintain the taxonomy. ${ }^{15}$

Since taxonomies are only structured lists of terms, the terms included in the taxonomy can strongly influence the taxonomy's usefulness. It is important to decide what morphological form to use in the taxonomy. Faith's linguistic analysis of taxonomy recommends avoiding terms such as company or brand names, organizational titles, or acronyms that are subject to sudden change or confusion; however, this recommendation is tempered by her acceptance of the principle that scope is the key factor in determining which terms belong in a taxonomy. ${ }^{16}$ Scope, in turn, is defined by the context of the taxonomy's intended users.

While user input is important in the taxonomy development process, basing the taxonomy on a limited group of users can restrict the taxonomy's utility in more diverse contexts. Alexander offers an approach to assess the process of decision-making in taxonomy creation projects that uses four criteria to characterize the taxonomy's objectivity and subjectivity. Those criteria are: openness to criticism, responsiveness to criticism, public accessibility of standards, and equality of intellectual authority. She uses the metaphor of "taxonomer as politician" to explain how taxonomy development balances these criteria to achieve objectivity, which Alexander characterizes as "open intersubjectivity." ${ }^{\text {"7 }}$ On platforms that support widespread collaboration, collaborative tagging, or user supplied tagging, can be used to generate a corpus of meaningful terms to serve as the basis for a more structured taxonomy; however, lack of guidelines and variation in background knowledge make it difficult to reuse collaboratively assigned tags. ${ }^{18}$ Although there are many approaches to taxonomy creation, most of the reports in this review stress the importance of user input in the taxonomy development process. There may not be a single best method of taxonomy creation; a taxonomy's usability and usefulness are ultimately determined by the users. 


\section{Costs and Benefits of Taxonomies}

Well-designed taxonomies can enable efficient retrieval of relevant information. Two important measures of information retrieval are precision (the percentage of relevant documents in a search result set) and recall (the amount of relevant documents in a result set expressed as a percentage of the total available number of relevant documents). It is not unusual for database searches to miss a great deal of relevant information (low recall) and to return a great deal of irrelevant information (low precision). Haig reports on group searches that ranged from capturing 6.5 to 19.6 percent of the available relevant documents, with most search result sets consisting of a small percentage of relevant papers, 6 to 30 percent. ${ }^{19}$ In contrast, Wang found recall scores ranging from 62.5 to 87.1 percent and precision scores ranging from 41.6 to 97.4 percent when evaluating the navigation effectiveness of a taxonomy for a library and information science school. ${ }^{20}$

Precise searches mean less time searching. High recall means more complete use of available information. In a paper highlighting the return on investment for taxonomy development, Ekionea and Swain align this capacity of taxonomy to increase efficient and effective use of resources with successful and sustainable business strategies. ${ }^{21}$ Classification can also play an important role in information retrieval systems designed to answer questions; it is the first step in the process of connecting information with a purpose. ${ }^{22}$ The utility of a taxonomy is not restricted to information retrieval. It can also perform an important role in knowledge transfer: browsing or studying a taxonomy can provide a user with subject knowledge, especially in the case of highly developed and specialized taxonomies. ${ }^{23}$

Although a fully developed and implemented taxonomy can be a timesaving resource for an organization, constructing taxonomies is very time consuming. It is common to consider using existing resources whenever possible; however, the specific context of an organization's purpose may not be reflected in an existing taxonomy. Haig et al. evaluated nine thesauruses related to medicine, education, and medical education and found them insufficient for describing medical education in the United Kingdom. ${ }^{24}$

Even when a taxonomy is established, its implementation may be very time consuming, particularly if consistency is a concern. In Park's taxonomy of weblogs, it took seventy hours of training to achieve acceptable interrater reliability among the seven coders using the taxonomy. ${ }^{25}$

\section{Method}

The taxonomy development team consisted of the organization's communications and assessment officers, the information management specialist, and the librarian consultant.
The team adopted Cisco's best practices for taxonomy development as the project's guidelines. The first step was to determine existing organizational strategies for accessing, organizing, and applying information. However, without a directly applicable example in the literature for obtaining the user input to meet this guideline, the information management specialist devised methods to solicit user input through staff interviews and an organization-wide survey, and conducted interviews with staff at organizations with a similar focus on reproductive health.

\section{Staff Interviews}

The communications and evaluations officers at IPPF/WHR selected ten staff members to represent a cross-section of roles, practices, and challenges in the areas of access to the literature, citation, and reference management. Staff members received meeting invitations and a short explanation of the needs assessment, and voluntarily participated in one-on-one interviews that lasted between thirty and fifty minutes. Interview questions probed for information about practices for finding, citing and tracking sources; determining trustworthiness; and sharing information with colleagues. The organization's communications officer vetted and edited the interview questions.

\section{Organization-wide Survey}

The survey goal was to understand the organization's need for access to subscription-based resources. The information management specialist obtained a list of thirty-five recommended publications through meetings and informal staff interviews. All staff members received a questionnaire requesting information about which of these recommended publications they subscribed and what publications they wanted to use but to which they lacked access. The information management specialist checked which of the desired journals were available either through the public library or as open access publications.

\section{Interviews with Staff at Related Organizations}

The communications and evaluation team suggested six related organizations to contact to get a sense of current practices, systems, and software used by organizations in the area of international sexual and reproductive health and rights. Interview questions focused on current citation management systems and institutional access to literature. Follow-up questions compared the effectiveness of approaches and explored recommendations for effective practice. Interviews lasted between twenty and forty minutes. 


\section{Results}

\section{Access to the Literature}

Staff had varying and extremely limited access to the literature. Many respondents reported encountering pay walls when trying to obtain needed papers. Staff members reported using the logins of interns, friends, or partners with a university library affiliation to access peer-reviewed papers. While no one expressed a desire to use peer-reviewed journals as their main source of data, many ranked them among their most trusted sources. Overall, these conditions limited staff knowledge of the literature in the field, as there was no centralized process for obtaining relevant papers.

\section{Citation and Data Management}

There was not a systematic procedure for documenting citations referenced in speeches, presentations, and publications. Staff members were often unable to locate source documents if they wanted to cite them later or if questions arose about the quality or accuracy of the source information. Without organization-wide citation practices, work shared with the public could not be consistently supported with references to evidence, which could potentially weaken the organization's credibility.

Data permeated every staff members' work and played crucial roles in both internal communications and how the staff represented the organization to its many stakeholders. Data use varied greatly by position and responsibilities and was reportedly used for reasons as varied as blog posts, speeches, statements to reporters, publications, conferences, papers, proposals, decision-making (strategy, focus, inclusion in proposals), reports, institutional proposals, and strategy papers. Five of the seven interviewees report that they used citations regularly for a variety of reasons: to confirm sources for their audience; to locate sources at a future date; to justify assertions; to provide context; to demonstrate need in the region; to maintain the organization's reputation; to identify strategies, need, evidence-based practices, or replicable models; to examine opportunities and challenges at regional and national levels; to track whether interventions are successful; and to provide increased transparency.

Many staff respondents reported a preference for data from prominent national and international organizations and felt that these organizations had already vetted the data and could serve as clearinghouses of trustworthy findings. Some interviewees suggested that, relative to peer-reviewed papers, these prominent national and international organizations had name recognition that instilled a sense of confidence in the data.

While many staff members reported saving some of their sources, they saved them in folders on their personal drive.
Others, who saved in a shared drive in team-wide resource folders, did not frequently utilize those repositories when they needed data. Wherever these sources were saved, they were not regularly updated and often became outdated. Descriptive file names were not common, which made searching and identifying content difficult. A third category of respondents did not save their sources and choose to repeat web searches when they needed to find a source of information again.

When staff members needed to share data or work across teams, they saw information management challenges manifest themselves. There was a lack of transparency between teams; many respondents reported that data regarding each program resided with the program officer; they did not know what information other teams possessed, and that lack of a formal system for sharing information was a problem. Most sharing of information and papers was through email, in conversations during meetings, or in the staff kitchen. Several respondents described the email channel as oversaturated and as a "black hole" for data. These conditions served to silo the different areas of work according to team/program and to limit the amount of potential cross-team and cross-program synergy.

\section{Practices at Related Organizations}

The information management specialist contacted three other large not-for-profit organizations that focus on women's health. Each organization had very different practices and capacities for citation management and access to the literature. Two of the three organizations had some form of library resources and used citation management software to draft reports and other publications. The need for citation management software and access to the literature was recognized and prioritized in organizations dedicated to producing academic papers and acting as a clearinghouse for the field. As an organization with a growing role in producing consumer health information, the organization that conducted this case study found validation for its interest in information management in the practices at other not-forprofits of comparable size and focus.

\section{Discussion}

\section{Information Management Pilot Development}

Following suggestions from the data management consultant, the organization implemented citation management software to provide a web-based shared library that enabled tagging, annotation, full document searches, collaborative PDF reading and mark-up, citation, and bibliography creation. The organization felt most comfortable with the customer support offered by a hosted service. These needs 


\begin{tabular}{ll}
\hline IGAPS in Mendely & Expanded IGAPS (Multi-level) \\
\hline - Information: CDC Reports & - Information \\
- Geography: United States & - Government Information \\
- Application: Fact Sheet & - CDC Reports \\
- Population: Latina & - Geography \\
- Subject: IUDs & - North America \\
& - United States \\
& - Application \\
& - Consumer Health Information \\
\hline
\end{tabular}

Figure 1. IGAPS Taxonomy Development

and preferences made Mendeley the best fit for the organization's information management pilot.

\section{IGAPS Taxonomy Development and Relationship to Taxonomy Literature}

The pilot development team held a series of meetings to determine the organization's priorities for organizing and using sources; it became apparent that important categories of information were related to the information's format, the geography of focus, the application for the information, the population to which the information relates, and the subject of the information. These categories were expressed using the mnemonic IGAPS (information, geography, application, population, and subject). Although there was interest in developing a hierarchical taxonomy of characteristics related to the IGAPS dimensions, the taxonomy was implemented within Mendeley, which would not support a taxonomy with a complex hierarchical structure at the time that the pilot was to be conducted (see figure 1).

Of the two restrictions proposed by Nickerson et al., the IGAPS taxonomy fits the collectively exhaustive restriction by requiring a characteristic for each one if its dimensions, but does not fit the mutually exclusive restriction in that it permits multiple characteristics in its subject dimension. Nickerson's restriction on mutual exclusivity would theoretically have helped efficient retrieval, but the organization's communications and assessment officers felt this would have made it difficult to classify all of a document's content with a single characteristic. The preference of the intended users for multiple labels for subject characteristic drove the departure from Nickerson's theoretical model. This departure from mutual exclusivity makes the IGAPS system a combination of faceted classification and descriptive metadata standard informed by user preference. While it is not a pure model, the pragmatic decision to defer to user preferences fits Cisco's best practice of keeping the context of the intended user in mind during taxonomy development. ${ }^{26}$

IGAPS is not unique as an approach to taxonomy that departs from Nickerson's mutual exclusivity criteria. In permitting the assignment of multiple characteristics to a single dimension, IGAPS was similar to Park's taxonomy used to classify weblogs. ${ }^{27}$ MeSH used by NLM also permits multiple subheadings that are not mutually exclusive. ${ }^{28}$ IGAPS followed Doria and IFLA in the effort to link the documents it classifies with user intent through its Action facet. ${ }^{29}$

With only five top-level categories, IGAPS conformed to the interpretation of Miller's rule suggested by Nickerson et al. and Neelameghan. ${ }^{30}$ This number of categories, and the faceted approach to analyzing documents, shared some similarity with Ranganathan's Personality, Matter, Energy, Space, Time (PMEST) colon classification system used in Indian libraries. ${ }^{31}$

The pilot development team edited a pre-made keyword guide for "resources related to family planning and reproductive health" and categorized it to fit into the IGAPS categories in an iterative process. ${ }^{32}$ This decision followed Cisco's best practice of incorporating existing taxonomic resources to save time; however, this taxonomy still required extensive editing to meet the needs of its intended users. ${ }^{33}$ Following Cisco, this editing was executed in an iterative process that incorporated input from the organization's stakeholders.

\section{Pilot Implementation}

IPPF/WHR lacked the budget to support a dedicated information/data manager who would assume responsibility for maintaining the taxonomy. Taking plans for taxonomy maintenance into account, the taxonomy was implemented using a decentralized model.

Each of the fifteen staff members participating in the pilot assigned taxonomic terms to documents that they deposited in the Mendeley group. The taxonomic terms were logged on a worksheet that was uploaded as an attachment to the record. The information management specialist transferred terms from the worksheet to the tag field in the record. A version of this worksheet is available in the appendix to this paper.

The worksheet listed the IGAPS categories and provided instructions about the descriptions of the scope of each category and instructions for assigning a range of one to five terms for each category. The worksheet included space for additional terms and questions. The pilot manager followed up on these entries with each staff participant and this input was incorporated into the taxonomy development.

\section{Pilot Assessment}

The practice of using data mostly from organizational reports and fact sheets, rather than academic papers, presented a 
challenge for easy integration of the pilot programs. Documents published by commonly used organizations often lacked accessible metadata that would allow automated creation of a complete Mendeley record. While the software allowed for making manual edits to records to complete metadata, this manual process was a barrier for staff use.

Staff contributions to the shared collection during the pilot period were not sufficient to create a robust organizational library. With only a few dozen items, it was not possible to test the utility of the IGAPS taxonomy for implementing more efficient searches, and the entire collection could be scanned at a glance. Staff members mentioned having limited time to spend with literature and expressed a desire for more time. The very limited growth of the library during the pilot period could be taken as an indicator that staff did not have much time to conduct literature searches and/or that staff literature searches were excessively time consuming. Qualitative interviews with the organization's leaders in communications and evaluation suggested that the pilot experience made valued contributions to their personal information management practices and their thinking about the organizational roles of information management and citation practices.

Overall, results of the pilot are mixed. Staff use of the IGAPS taxonomy during the pilot was inconsistent. During the pilot, the information management specialist was able to correct inconsistent uses of IGAPS; however, without dedicated staff to oversee the application of IGAPS to documents added to the Mendeley repository, it was clear that the information management system envisioned by the taxonomy development team was not sustainable. Despite this shortcoming, the pilot produced some valued outcomes: 1) it established staff use of Mendeley as a citation management system and organizational repository; 2) it delivered the IGAPS taxonomy as an organizational document; and 3) it provided staff with citation training.

\section{Recommendations}

User-generated libraries are challenging, even with a dedicated staff to curate them. Organizations without permanent librarians or information management specialists may face challenges when establishing a new information management system. This paper describes the beginning of a process that would require organizational culture shifts and investment of resources to effect sustainable change. Longterm success would depend on progress and development in three interrelated areas: staff commitment, culture shift, and training.

\section{Staff Commitment}

Giving staff an incentive to participate is critical to the long-term success of information management projects. Staff members must be informed and reminded that it is important to make the organizational library a part of their work plan, that this is a significant way to grow as an organization, and especially that it will benefit them as employees.

\section{Culture Shift}

The origin of changes to organizational information management practices lies in the desire for a shift in organizational culture. Getting staff to place greater value on tracking and citing sources will lead to an increase in the integrity of information-used both internally and externally-and reduce frustration and time spent backtracking statistics. Citation training was included in the pilot described in this report as a first step towards this culture shift.

\section{Training}

Continuing software and methods training is necessary, even for those who gained experience during pilot programs. These training sessions might be refreshers, updates on new features, additional ways to use software, opportunities to ask questions or raise technical issues, etc. It is important to share ideas about how to incorporate new software into daily routines, how each individual's engagement impacts the utility of the library, etc. A taxonomy is not useful if staff do not apply it in their information storage and retrieval practices. Staff need repeated training sessions to inculcate the best practices for applying the taxonomy in tagging to searching. Participants should also be updated when the taxonomy changes. As the project progresses, training on points of access and search methods and best citation practices will be necessary. These long-term considerations form a key framework that should continue to be discussed as steps are taken to address reference management and access to literature within a not-for-profit organization.

\section{References}

1. Robert C. Nickerson, Upkar Varshney, and Jan Muntermann, "A Method for Taxonomy Development and Its Application in Information Systems," European Journal of Information Systems 22 , no. 3 (2013): 336-59.

2. Ibid.

3. A. Neelameghan, "Seminal Mnemonics in Knowledge Organization: Ancient Traditions and Modern Practices," Information Studies 12, no. 1 (2006): 5-26.

4. Daniel CannCasciato, "Ethical Considerations in Classification Practice: A Case Study Using Creationism and Intelligent Design.," Cataloging \& Classification Quarterly 49, no. 5 (June 2011): 408-27.

5. Earl H. McKinney Jr., J. Charles, and I. I. Yoos, "Information 
About Information: A Taxonomy of Views," MIS Quarterly 34, no. 2 (2010): 329-344.

6. David L. Hull, "Bias and Commitment in Science: Phenetics and Cladistics," Annals of Science 42, no. 3 (985): 319-38, https://doi.org/10.1080/00033798500200231.

7. Lutz Bornmann, "Is There Currently a Scientific Revolution in Scientometrics?," Journal of the Association for Information Science \& Technology 65, no. 3 (2014): 647-48, https:// doi.org/10.1002/asi.23073.

8. Sung-Yeon Park et al., "Inside the Blogosphere: A Taxonomy and Framing Analysis of Abortion Weblogs," Social Science Journal 50, no. 4 (2013): 616-24, https://doi.org/10.1016/j.sos cij.2013.04.014.

9. Natalie Jomini Stroud, Ashley Muddiman, and Jae Kook Lee, "Seeing Media as Group Members: An Evaluation of Partisan Bias Perceptions," Journal of Communication 64, no. 5 (2014): 874-94, https://doi.org/10.1111/jcom.12110.

10. Sergio Tezanos Vázquez and Andy Sumner, "Revisiting the Meaning of Development: A Multidimensional Taxonomy of Developing Countries," Journal of Development Studies 49, no. 12 (2013): 1728-45, https://doi.org/10.1080/00220388.20 13.822071 .

11. Celia Jenkins, Charles Oppenheim, and Steve Probets, "RoMEO Studies 7: Creation of a Controlled Vocabulary to Analyse Copyright Transfer Agreements, Journal of Information Science 34, no. 3 (2008): 290-307.

12. Orélie Desfriches Doria, "The Role of Activities Awareness in Faceted Classification Development," Knowledge Organization 39, no. 4 (2012): 283-91.

13. Nickerson, Varshney, and Muntermann, "A Method for Taxonomy Development."

14. IFLA Study Group on the Functional Requirements for Bibliographic Records, "Functional Requirements for Bibliographic Records," Final Report, IFLA Series on Bibliographic Control (Munich, 1998), accessed August 8, 2015, http:// www.ifla.org/files/assets/cataloguing/frbr/frbr_2008.pdf.

15. Susan Cisco and Wanda Jackson, "Creating Order out of Chaos with Taxonomies," Information Management Journal 39, no. 3 (2005): 44-50.

16. Ashleigh Faith, "Linguistic Analysis of Taxonomy Facet Creation and Validation," Key Words 21, no. 1 (2013): 11-15.

17. Fran Alexander, "Devising a Framework for Assessing the Subjectivity and Objectivity of Information Taxonomy Projects," Journal of Documentation 70, no. 1 (2014): 4-24, https://doi.org/10.1108/JD-09-2012-0117.

18. Eric Charton et al., "Using Collaborative Tagging for Text Classification: From Text Classification to Opinion Mining,"
Informatics 1, no. 1 (2013): 32-51, https://doi.org/10.3390 /informatics1010032.

19. Alex Haig et al., "METRO_the Creation of a Taxonomy for Medical Education," Health Information \& Libraries Journal 21, no. 4 (2004): 211-19.

20. Zhonghong Wang, Christopher S.G. Khoo, and Abdus Sattar Chaudhry, "Evaluation of the Navigation Effectiveness of an Organizational Taxonomy Built on a General Classification Scheme and Domain Thesauri," Journal of the Association for Information Science \& Technology 65, no. 5 (2014): 94863, https://doi.org/10.1002/asi.23017.

21. Jean-Pierre Ekionea and Deborah Swain, "Developing and Aligning a Knowledge Management Strategy: Towards a Taxonomy and a Framework," International Journal of Knowledge Management 4, no. 1 (2008): 29-45.

22. Maheen Bakhtyar et al., "Creating Multi-Level Class Hierarchy for Question Classification with NP Analysis and WordNet," Journal of Digital Information Management 10, no. 6 (2012): 379-88.

23. Cisco and Jackson, "Creating Order out of Chaos with Taxonomies."

24. Haig et al., "METRO — the Creation of a Taxonomy for Medical Education."

25. Park et al., "Inside the Blogosphere."

26. Cisco and Jackson, "Creating Order out of Chaos with Taxonomies".

27. Park et al., "Inside the Blogosphere."

28. "Introduction: What Is MeSH?," Training Material and Manuals, US National Library of Medicine, accessed January 21, 2014, http://www.nlm.nih.gov/bsd/disted/meshtutorial/intro duction/index.html.

29. Doria, "The Role of Activities Awareness in Faceted Classification Development."

30. Nickerson, Varshney, and Muntermann, "A Method for Taxonomy Development”; Neelameghan, “Seminal Mnemonics in Knowledge Organization: Ancient Traditions and Modern Practices."

31. S. R. Ranganathan, Colon Classification: Basic Classification (Ess Ess Publications, 2007).

32. Knowledge for Health Project, "A User's Guide to POPLINE Keywords" (Johns Hopkins Center for Communication Programs, 2014), accessed August 11, 2015, http://www.popline .org/sites/default/files/KWGuide_10thEd.pdf.

33. Cisco and Jackson, "Creating Order out of Chaos with Taxonomies." 


\section{Appendix. IGAPS Worksheet}

Refer to the taxonomy while you're reviewing the document-flipping back and forth between the article and the taxonomy-to remind yourself of IGAPS categories while reading.

Select tags for each category of IGAPS while reading. Adhere to the following rules:

1. None of the IGAPS categories should have more than five tags.

2. Be concise by using few tags as possible. Focus on the overarching themes of the article.

3. Each category of IGAPS should have at least one tag.

4. Use the terms closest to the language used in the article.

IGAPS Term selections:

Information:

(What type of information is this document?)

\section{Geography:}

(What country and/or region does this document focus on?)

Application:

(How will this information be applied?)

\section{Population:}

(What groups does this document focus on?)

\section{Subject:}

(What are the main themes that appear in this document?)

\section{Additional Comments:}

